\title{
LA RESISTENCIA AL TIZÓN TARDÍO COMO HERRAMIENTA DE CONTROL EN LOS PAÍSES EN DESARROLLO ${ }^{1}$
}

\author{
G. A. Forbes ${ }^{2}$; M. Huarte ${ }^{3}$
}

Manuscrito recibido: $21 / 09 / 2014$

Aceptado: 12/11/2014

Disponible en línea: Diciembre 2014

\section{Resumen}

El tizón tardío de la papa puede ser controlado con varias estrategiass, incluyendo la resistencia del huesped. Desafortunadamente, los esfuerzos hasta la fecha para utilizar la resistencia del huésped han llevado a un éxito limitado y también al fracaso de promover la generaración de poblaciones del patógeno que han evolucionado rápidamente para superar la resistencia o a la decepción provocada por la limitada adopción de los pocos cultivares con resistencia duradera. Varios problemas dificultan la difusión de cultivares resistentes, incluyendo a las poblaciones del patógeno que evolucionan rápidamente, las preferencias del mercado, la asociación de la resistencia con maduración tardía, y cuestiones metodológicas, como el fenotipado inexacto y la comunicación insuficiente entre los investigadores, esto último sobre todo en los países en desarrollo. Nuevas tecnologías y enfoques que mejoren la identificación y adopción de cultivares resistentes son discutidas, incluidos la biotecnología, el perfeccionamiento del mejoramiento convencional, el monitoreo de patógenos, un fenotipado más preciso y una mejor colaboración en la investigación.

Palabras claves adicionales: países en desarrollo, resistencia del huesped, tizón tardío, papa

(1) Este artículo de revisión es una traducción ampliada de Forbes, 2012. (2) Autor de correspondencia. Centro Internacional de la Papa, Beijing, China e-mail: g.forbes@cgiar.org;

(3) EEA INTA Balcarce, Buenos Aires, Argentina 


\title{
LATE BLIGHT RESISTANCE AS A CONTROL TOOL IN DEVELOPING COUNTRIES
}

\begin{abstract}
Summary
Potato late blight may be controlled by several approaches including host resistance. Unfortunately, efforts to date to use host resistance have led to limited successes and many failures as pathogen populations have rapidly evolved to overcome resistance or durably resistant cultivars have had limited adoption. Several problems hinder diffusion of resistant cultivars, including a rapidly evolving pathogen population, market preferences, association of resistance with late maturation, and methodological issues such as inaccurate phenotyping and insufficient communication among researchers, the latter particularly in developing countries. New technologies and approaches that improve the identification and adoption of resistant cultivars are discussed, including biotechnology, enhanced conventional breeding, pathogen monitoring, improved phenotyping, and better research collaboration.
\end{abstract}

Additional keywords: developing countries, host resistance, late blight, potato

\section{Introducción}

En los países en desarrollo, varias enfermedades reducen constantemente la productividad de la papa, dando lugar a una gran brecha entre los rendimientos alcanzables y los reales y, a menudo, con consecuencias sobre la salud y ocasionando riesgos ambientales debido al uso excesivo de pesticidas. Una de las más importantes de estas enfermedades es el Tizón Tardío de la Papa (TTP) causado por el oomiceto patógeno Phytophthora infestans. Se ha estimado que el TTP puede costar hasta mil millones de euros anuales incluyendo tanto las pérdidas de rendimiento como el costo de los fungicidas y su aplicación (Haverkort et al., 2009). Esta cifra no incluye costos emergentes de los riesgos ambientales y a la salud. El TTP está presente en todas las principales zonas donde se cultiva la papa (Hijmans et al., 2000), 
dependiendo la intensidad de la enfermedad de las condiciones climáticas (temperatura moderada y largos períodos de humedad de la hoja). En algunas áreas, tales como las tierras altas tropicales, el TTP es particularmente perjudicial, ya que coincide con la producción de papa durante todo el año (Garrett et al., 2001) y la baja capacidad de los agricultores para entender y manejar la enfermedad (Andrade-Piedra et al., 2009). Muchas zonas donde afecta el TTP en el mundo en desarrollo son caracterizadas por la pobreza y la inseguridad alimentaria, por lo que la enfermedad es una grave amenaza para los productores de papa en pequeña escala. El TTP generalmente puede ser controlado efectivamente con fungicidas; sin embargo, muchos de éstos pueden plantear riesgos ambientales o de salud (Kromann et al. 2011), en particular en países en desarrollo donde los agricultores asperjan manualmente y rara vez o nunca usan ropa de protección (Orozco et al., 2009). Para encontrar soluciones sostenibles al problema TTP, los mejoradores de los países en desarrollo han luchado durante décadas para desarrollar cultivares de papa que tiengan altos niveles de resistencia a $P$. infestans, mientras que al mismo tiempo cumplan con las numerosas demandas de la cadena productiva de la papa (Forbes; Landeo, 2006). Este esfuerzo ha dado lugar sólo a un éxito parcial; algunas razones para ello, así como los pasos que se pueden tomar para mayor éxito se discuten a continuación.

El Mejoramiento para la Resistencia al Tizón Tardío de la Papa y la dinámica de la renovación de cultivares

La papa fue introducida desde América del Sur a Europa en la segunda mitad del siglo XVI y posteriormente se extendió a nivel mundial. La adopción de la papa fue lenta en Europa, aunque el cultivo finalmente se convirtió en un elemento básico y contribuyó al crecimiento de la población en el siglo XIX (Salaman, 1989). El patógeno que causa el TTP se introdujo 
en América del Norte y Europa hacia 1840 y esto llevó a las grandes epidemias de enfermedades con consecuencias humanas terribles (Bourke, 1993). El patógeno se extendió posteriormente en todo el mundo, presumiblemente con el comercio de papa semilla (Fry, 2008). Así, la introducción del patógeno en América del Norte, Europa y luego a nivel mundial, seguió a la introducción de la papa en nuevas regiones durante varios siglos. Por lo tanto, en particular en Europa y, en diferentes grados, en otros lugares fuera de América del Sur, el cultivo fue creciendo durante períodos variables sin amenaza del TTP al inicio de la expansión del cultivo.

Nunca ha habido una escala universalmente disponible para medir con precisión la resistencia en papa al TTP, y por lo tanto, no es fácil determinar el nivel de resistencia en los cultivares que se utilizaron después que el TTP se introdujo en Norte América y Europa. No obstante, los informes de los cultivares de papa con resistencia a TTP se remontan a la década de 1800, y al parecer, el mejoramiento era una actividad importante en Irlanda durante las décadas que siguieron a la hambruna (Bourke, 1993). Un número de cultivares con algún nivel de resistencia a TTP estaban disponibles en Europa a finales de 1800 (Umaerus et al., 1983). Es posible que las papas cultivadas en Europa en la era post-hambruna tenían niveles apreciables de resistencia a TTP; al menos un autor ha sugerido que los esfuerzos por mejorar y seleccionar para la resistencia fueron socavados por el descubrimiento del Caldo Bordelés, en la década de 1880, que dio a los agricultores la oportunidad para plantar cultivares susceptibles pero que tenían otras características deseables (Robinson, 1996).

Existe un debate en curso sobre: el tiempo y el mecanismo de la aparición del TTP en América del Sur, el origen histórico de la papa, la coevolución del hongo y la papa y la región donde el cultivo se ha cultivado por más tiempo (Gómez-Alpízar et al., 2007, Micheletto et al.,. 2000). Por lo tanto, cualquier especulación sobre el papel histórico de la resistencia 
genética a esta enfermedad en América del Sur es de poca utilidad hasta que la historia de la enfermedad en esta región se conozca con mayor certeza.

Independientemente de los niveles de resistencia en papa en las décadas posteriores a la hambruna, la mayoría de los cultivares de papa cultivados actualmente en todo el mundo son susceptibles al TTP. En los países industrializados, los agricultores prefieren el costo marginal del uso de fungicidas en comparación al riesgo percibido de usar un cultivar resistente (Cooke et al., 2011). También existe la presión de los procesadores y las multinacionales para utilizar cultivares específicos pero susceptibles al TTP, tales como Russet Burbank. En los países en desarrollo, donde la enfermedad es pobremente manejada por muchas razones (por ejemplo, la alta presión de la enfermedad, los problemas de acceso a los fungicidas y el desconocimiento de los agricultores de la dinámica de la enfermedad), cabría esperar que la resistencia del huésped desempeñara un papel importante en el manejo de la enfermedad, y de hecho, se puede argumentar que, en promedio, los cultivares de los países en desarrollo tienen niveles más altos de resistencia (Forbes et al., 2005). En Kenia, por ejemplo, el 45,4\% del área de producción de papa está cubierta con cultivares que tienen una escala de resistencia ponderada de 7,14, y el 54,6\% de la zona de producción de papa de Uganda está cubierta por el cultivar Victoria, que tiene un valor de escala de 6.26 (Tabla 1). Este relativamente pequeño nivel de resistencia puede no parecer relevante para el manejo de la enfermedad, pero muchos de estos cultivares son conocidos por los agricultores como más resistentes al TTP que otros cultivares disponibles (Nyankanga et al., 2004). En Ruanda, el 20,8\% de la zona de producción de papa se planta con Cruza 148, que tiene un valor de escala de 2 y por lo tanto es altamente resistente a TTP.

Grandes áreas de la producción de papa en muchos países en desarrollo son plantadas con cultivares altamente susceptibles que tendrían valores de 8 o 9 (altamente susceptibles) en la escala de Yuen y Forbes (2009). Por ejemplo, el cultivar más popular en Indonesia, que cubre 
aproximadamente $80 \%$ de la superficie, es Granola, que es altamente susceptible a TTP. Entonces surge la pregunta, ¿por qué no ha habido más éxito en el desarrollo, la difusión y adopción de cultivares resistentes, sobre todo en los países en desarrollo?

Hay varias razones por las que los cultivares de papa susceptibles al TTP están siendo ampliamente cultivados. En un estudio realizado en Cajamarca, Perú, los productores entrevistados indicaron que el TTP era el principal problema en la zona, el cual reducía la producción significativamente. La mayoría de los entrevistados identificaron claramente las condiciones climáticas que favorecen la enfermedad; pero no conocían el agente causal de la enfermedad ni tenían la habilidad para diagnosticarla correctamente. La mayoría de agricultores consideraron al control químico como la principal práctica de control y usaron 6.6 aplicaciones en promedio. En menor medida se mencionó el uso de variedades resistentes y de diferentes fechas de plantación (Ortiz et al., 1999).

Al igual que con muchos de los cultivos, la cadena de comercialización de la papa prioriza otras cualidades y un cultivar que no tiene resistencia a una enfermedad puede ser seleccionado por otros atributos, asi, el cultivar resistente a TTP Cruza 148 (Tabla 1), una vez que se cultivó ampliamente en el Zona del Lago Kivu en África, ha perdido terreno frente a los cultivares más susceptibles que logran un valor más alto en el mercado. Los cultivares que han reemplazado a Cruza 148, como Asante (Tabla 1), tienen un tamaño de tubérculo y forma más uniformes. Cruza 148 también posee un anillo vascular pigmentado, que resulta poco atractivo a los consumidores locales. El caso del cultivar Dunja, de origen alemán y con reconocido nivel de resistencia al TTP, fue descartado en Argentina por razones de tipo culinario y de aspecto de tubérculo. La demanda del mercado es un factor que influye en el volumen de negocios y con frecuencia limita el retorno económico de un cultivar. El cultivar europeo, Granola, se ha arraigado en el sistema de producción de Indonesia aunque a menudo se pulveriza más de 20 veces por temporada para controlar el TTP. Esta situación también se 
da en Centro América y Venezuela donde se cultiva Granola y el TTP es muy importante. A pesar de estos ejemplos, el efecto del mercado sobre el margen de ganancias de un cultivar es más fuerte en el mundo industrializado, donde los componentes del mercado se han vuelto altamente especializados (Walker et al.,1999); por ello, la adopción de cultivares resistentes en los países en desarrollo aún sigue teniendo oportunidad. Otra razón para el uso generalizado de cultivares susceptibles es que la resistencia a la enfermedad no ha sido estable; muchos cultivares liberados como resistentes al TTP se han convertido rápidamente en susceptibles a medida que evoluciona la población del patógeno (Tabla 2). El cultivar Victoria en Uganda (conocido como Asante en Kenya) fue lanzado como muy resistente a la enfermedad, pero ha "caído" a una susceptibilidad aparentemente estable con un nivel de aproximadamente 6 (Tabla 1) y se cultiva ampliamente. Algunos investigadores han encontrado que los genes R "derrotados" se asocian con resistencia parcial (Stewart et al., 2003). Por ello, la naturaleza efímera de la resistencia mediada por genes $\mathrm{R}$ y la dificultad de transferir la resistencia heredada cuantitativamente han tornado muy difícil la tarea de identificación y difusión de la resistencia duradera a TTP (Cooke et al., 2011).

Históricamente, el mejoramiento para resistencia al TTP en la papa no fue siempre considerado como una via muerta. De hecho, el entusiasmo por la resistencia de la planta huésped al TTP surgió en la década de 1950 cuando muy altos niveles de resistencia fueron encontrados en las especies silvestres en México, particularmente en la especie Solanum demissum (Wastie, 1991). Esta resistencia, denominada raza específica, de genes $\mathrm{R}$ o cualitativa, fue conferida por genes individuales y era por lo tanto fácil de incorporar en nuevos cultivares. Rápidamente se comenzó a transferir esta resistencia a la papa cultivada, pero desafortunadamente, la pérdida de resistencia debido a la evolución del patógeno ocurrió de forma concomitante. Por la década de 1960, el ciclo de auge y caída de esta resistencia 
raza-específica se convirtió en uno de los modelos sobre la cual JE Vanderplank desarrolló los fundamentos de la epidemiología de las enfermedades de plantas. 
Tabla 1. Niveles de resistencia foliar a Phytophthora infestans de algunos cultivares de papa más cultivadas en Kenia y otros países de Africa.

\begin{tabular}{|c|c|c|c|c|c|}
\hline Cultivar & $\begin{array}{|lr|}\text { Año } & \text { de } \\
\text { lanzamiento }\end{array}$ & Área (ha) & Área $(\%)^{\mathrm{a}}$ & Escala $^{b}$ & $\begin{array}{l}\text { Escala } \\
\text { ponderada }\end{array}$ \\
\hline \multicolumn{6}{|l|}{ Kenya } \\
\hline Tigoni & 1998 & 25.550 & 16,7 & 6,26 & 2,30 \\
\hline Asante & 1998 & 9639 & 6,3 & 6,46 & 0,90 \\
\hline Purple Tigonid & $?$ & 5355 & 3.5 & 8.99 & 0.69 \\
\hline Dutch Robijn & 1945 & 6732 & 4,4 & 7,54 & 0,73 \\
\hline Thima Thuti $^{\mathrm{d}}$ & ? & 17.441 & 11,4 & 8,11 & 2,04 \\
\hline Shangid & ? & 4,743 & 3,1 & 7,05 & 0,48 \\
\hline Total / average $\mathrm{e}^{\mathrm{e}}$ & & 69.460 & 45,4 & 7,14 & \\
\hline Etiopía-Jalene & 2002 & 22.390 & 13,6 & 5,38 & \\
\hline Ruanda-Cruza148 & 1981 & 31.362 & 20.8 & 2 & \\
\hline $\begin{array}{l}\text { Uganda-Victoria } \\
(=\text { Asante })^{\mathrm{f}}\end{array}$ & \begin{tabular}{|l|}
1.988 \\
\end{tabular} & 54.672 & 53,6 & 6,46 & \\
\hline
\end{tabular}

${ }^{\text {a }}$ Porcentaje de la producción total del país

${ }^{\mathrm{b}}$ Yuen y Forbes (2009); los valores aumentan con el aumento de los niveles de la enfermedad, y por lo tanto, la referencia es a la susceptibilidad en lugar de la resistencia (los números más altos son más susceptibles); un cultivar altamente susceptible tendría un valor de 9. Los datos de campo para los valores de la escala de todos los cultivares, excepto Cruza 148, proceden de ensayos realizados por el Centro Internacional de la Papa en Kenia (datos no publicados); los datos para Cruza 148 provienen de Forbes et al. (2005)

${ }^{c}$ Los valores de escala ponderada se forman a partir del porcentaje de área de cada cultivar de Kenia en relación con el área de la producción total de todos los cultivares en Kenia; como ejemplo, para Tigoni, este se calcula como $(25550 / 69460)$ x 6.26. Esto da una estimación del valor promedio de la escala sobre el área ocupada por estos cultivares.

${ }^{\mathrm{d}}$ Origen incierto

${ }^{\mathrm{e}}$ Total de cultivares listados; escala media ponderada para los cultivares listados; véase la nota ${ }^{\mathrm{c}}$

${ }^{\mathrm{f}}$ Este cultivar fue lanzado por primera vez en Uganda como Victoria y posteriormente en Kenia como Asante.

La identificación, utilización y fracaso de la resistencia raza- específica derivada de $S$. demissum contrastó con la resistencia que se encuentra en algunos cultivares que, aunque no completa parecería ser durable (Vanderplank, 1956). Esta dicotomía fenotípica evidente en los tipos de resistencia llevó a décadas de investigación sobre la naturaleza de la resistencia del huésped a $P$. infestans que continúa hoy con toda su fuerza (Nowicki et al., 2012). El fracaso de la resistencia proveniente de $S$. demissum para proporcionar protección a campo también condujo a un cambio en el énfasis por parte de los mejoradores hacia la resistencia parcial, heredada cuantitativamente (a menudo referida como horizontal, de campo o resistencia cuantitativa) (Bradshaw et al., 1995). Estrada et al. (1994) mencionan la existencia de algunos cultivares que mantienen su resistencia "parcial" por varios años, como es el caso de 
Monserrate (Colombia), Santa Catalina (Ecuador), Perricholi/Runa Toralapa (Perú/Bolivia), Ackersegen (Alemania), Record, Pimpernel y Libertas (Holanda) y Atzimba (México). Ya en 1997 Bolivia disponía de cultivares con resistencia parcial como Jaspe y Robusta, aún hoy presentes en el campo de los agricultores y que poseían germoplasma de origen colombiano (Carrasco et al, 1997). En ese mismo país se cuenta con manejo integrado de cultivo, combinando una variedad resistente como Robusta y uso racional de fungicidas (Navia et al, 2001). CIP inició un programa en la década de 1990 para identificar cultivares con resistencia que no fuera conferida por los genes R conocidos de $S$. demissum seleccionando poblaciones segregantes de papa con razas del patógeno que superaban todos los genes $\mathrm{R}$ conocidos de dicha especie (Forbes; Landeo, 2006). Muchos países en desarrollo se beneficiaron de este programa; a modo de ejemplo se puede mencionar a Panamá, donde IDIAP92 alcanzó difusión por requerir un $50 \%$ menos de aplicaciones fungicidas que Granola y Amigo (Morales, 2001).

El mejoramiento para un caracter cuantitativo es difícil, y en este caso en particular, la resistencia al TTP era a menudo asociada con la madurez tardía y con la sensibilidad longitud del día (Visker, 2005). Un número de cultivares con resistencia cuantitativa al TTP aparentemente estable, ha sido liberado en los países en desarrollo (Tabla 2; Cooke et al., 2011; Grünwald et al., 2002), pero no muchos se cultivan en gran superficie o tienen sólo niveles moderados de resistencia. Existen numerosas citas sobre evaluación de la resistencia en varios países de Latinoamérica, con las carácterísticas ya mencionadas (Gabriel et al, 2012). 
Tabla 2 Niveles actuales de resistencia foliar a Phytophthora infestans de algunos cultivares de papa originalmente lanzados como altamente resistentes.

\begin{tabular}{|l|c|c|c|}
\hline Cultivar & $\begin{array}{c}\text { Año de } \\
\text { lanzamiento }\end{array}$ & País & $\begin{array}{c}\text { Escala de } \\
\text { Susceptibilidad }\end{array}$ \\
\hline Arka $^{\mathrm{b}}$ & 1980 & Tanzania & 9 \\
\hline Capiro $^{\mathrm{c}}$ & 1968 & Colombia & 9 \\
\hline Cecelia $^{\mathrm{c}}$ & 1962 & Ecuador & 8 \\
\hline Gabriela $^{\mathrm{c}}$ & 1982 & Ecuador & 8 \\
\hline Superchola $^{\mathrm{c}}$ & $?$ & Ecuador & 8 \\
\hline Canchan $^{\mathrm{d}}$ & 1990 & Perú & 9 \\
\hline Amarilis $^{\mathrm{d}}$ & 1993 & Perú & 7 \\
\hline Pampeana INTA $^{\mathrm{e}}$ & 1985 & Argentina & 6 \\
\hline
\end{tabular}

${ }^{a}$ Yuen y Forbes (2009); los valores aumentan con el aumento de los niveles de la enfermedad, y por lo tanto, la referencia es referida a susceptibilidad en lugar de resistencia (es decir, los números más altos son más susceptibles); un cultivar altamente susceptible tendría un valor de 9

${ }^{\mathrm{b}}$ Lanzado por primera vez en Malawi; información basada en encuestas realizadas por el Centro Internacional de la Papa (CIP) (datos no publicados); datos de resistencia de los ensayos realizados por el CIP en Kenia (inédito)

${ }^{\mathrm{c}}$ Los datos de los ensayos realizados por el CIP en Ecuador (no publicado)

${ }^{\mathrm{d}}$ Los datos de los ensayos realizados por el CIP en el Perú (inédito)

${ }^{\mathrm{e}}$ Adapted from Trognitz et al 2001

En relación con muchos otros cultivos, la difusión de nuevos cultivares de papa es un proceso lento y difícil debido a la baja tasa de multiplicación, la naturaleza clonal, la voluminosidad y el carácter perecedero de los tubérculos. En los países industrializados, hay empresas de producción de semillas altamente desarrolladas que pueden multiplicar y distribuir rápidamente un nuevo cultivar. Estos sistemas no existen o están poco desarrollados en los países en desarrollo y los nuevos cultivares generalmente se propagan de un agricultor a otro, que es un proceso mucho más lento. Esta propagación de agricultor a agricultor puede llevar rápidamente a una reducción en el estado sanitario de la papa semilla, que también podría afectar a la adopción del nuevo cultivar. Pareciera que los cultivares que pueden "despegar" por su cuenta, frecuentemente tienen algún nivel de resistencia a uno o más de los virus importantes de la papa, un ejemplo de esto es el cultivar chino Cooperación 88 (Li et al., 2010) y el cultivar Achirana INTA (CIP 24) en ese mismo país (Bofu et al., 1996).

Estrategias para acelerar el desarrollo y adopción de cultivares con resistencia duradera al TTP 
El mejoramiento convencional para la resistencia al TTP ha producido algunos éxitos, y aunque no siempre ampliamente cultivados, hay un número de cultivares en los países en desarrollo y en los países industrializados con niveles mensurables de resistencia (Forbes et al., 2005; Cooke et al,. 2011). Con el advenimiento de la nueva tecnología molecular y la secuenciación de los genomas del patógeno y del huesped, ahora hay más herramientas para desentrañar los misterios asociados con la resistencia al TTP, particularmente los factores limitantes a la durabilidad de la misma. Una estrategia para aumentar la probabilidad de encontrar una resistencia duradera es ampliar la base genética de la resistencia. Una alternativa es el mejor uso del germoplasma silvestre con búsquedas dirigidas de nuevas fuentes de resistencia. Se utilizó un análisis filogenético molecular para identificar un clado (Piurana) que no había sido utilizado como fuente de resistencia. Un importante locus de resistencia cuantitativo fue identificado en el cromosoma 11 de la especie S. paucissectum de este clado, recientemente caracterizado (Villamón et al., 2005). El mapeo genético comparativo de fuentes de resistencia disponibles y de nuevas fuentes puede idealmente conducir a la utilización estratégica de germoplasma facilitada por marcadores moleculares. Algunos científicos han abogado por una búsqueda de nuevos genes R que es de esperar sean de "amplio espectro" (es decir, eficaces contra la mayoría o, potencialmente, la totalidad de la población del patógeno), particularmente de la especie silvestre S. bulbocastanum. Sin embargo pareciera que el potencial evolutivo del patógeno es similar para estos nuevos genes como lo fue para los genes de S. demissum (Champouret et al., 2009;. Tan et al., 2010). Estos genes "nuevos" se han introgresado a través del mejoramiento convencional o la ingeniería genética en cultivares de papa existentes (Lammerts van Bueren et al., 2008.; Haverkort et al., 2009) y podrían, en teoría, ya sea desplegarse en múltiples líneas o por "piramidizado" en un solo genotipo. Suponiendo que la frecuencia de patogenicidad para cada gen fuera baja en la población del patógeno, los aislamientos patogénicos para el "cassete" completo serían 
extremadamente raros y esto podría significar que la resistencia sería duradera. Para las infraestructuras agrícolas desarrolladas, donde existen buenos sistemas de producción de semilla y los cultivares se pueden cambiar con facilidad, el despliegue estratégico de estos genes en espacio y tiempo también puede ser posible (Tan et al., 2010). Aunque, en realidad la implementación del despliegue de este tipo de genes sería difícil, ya que requiere la previsión de la demanda futura de semilla y varios años para hacer volumen de una nueva cultivar. Para los países en desarrollo, donde los sistemas formales y estructurados de semillas no están presentes en gran medida, la idea del despliegue de genes es irrelevante y los cultivares resistentes deben probar su resistencia duradera tanto espacial como temporalmente. Ya sea que el mejoramiento de cultivos sea implementado por mejoramiento convencional o por medio de modificación genética, es importante lograr un mayor conocimiento de las fuentes de resistencia. Para ese fin, un esfuerzo internacional para identificar genes $\mathrm{R}$ ha llevado al desarrollo de una base de datos de fuentes de resistencia (SolRgenes), alojada en la Universidad de Wageningen (Vleeshouwers et al., 2011).

\section{Monitoreo de Patógenos}

El genoma de $P$. infestans se ha secuenciado recientemente y presenta un arsenal muy variado de genes efectores (necesarios para causar la enfermedad), que están evolucionando más rápidamente que los genes normales. Este descubrimiento llevó a los investigadores a postular una "velocidad dual" para el genoma del patógeno (Haas et al., 2009). En reconocimiento de la importancia de comprender la dinámica de población de este patógeno, se han desarrollado un conjunto de marcadores para la caracterización genotípica y fenotípica de los aislamientos (Cooke; Lees, 2004). Los esfuerzos para obtener niveles nuevos y duraderos de resistencia, descritos anteriormente, se podrían mejorar mediante el monitoreo de las poblaciones del patógeno con estos marcadores y, finalmente, el desarrollo de otros marcadores más precisos. 
El cambio en el patógeno puede estar relacionado con la pérdida de eficacia de los fungicidas (Cooke; Lees. 2004) y también por un cambio radical en el proceso de la enfermedad debido a la participación de oosporas en el inicio de la enfermedad (Cooke et al., 2011). Durante la iniciación de la enfermedad a principios de cada nueva temporada, la acción de las oosporas tiende a afectar a todos los cultivares de papa, pero podría, sin embargo, afectar también a la interpretación de los resultados del tamizado por resistencia si no es detectada. La reproducción sexual, con la producción de oosporas, también conduce a una mayor diversidad en la población del patógeno (Yuen, 2012).

\section{Mejora del fenotipado y del manejo de datos con mayor coordinación de la investigación}

Los investigadores de la papa han tendido a utilizar mucho las clasificaciones subjetivas, del tipo "resistente", "moderadamente resistente", y "susceptible". Estas clasificaciones semicuantitativas son inapropiadas para incluso la más simple de las operaciones matemáticas y por lo tanto proporcionan información limitada acerca de la resistencia del huésped. Para superar el problema de una mala caracterización de la resistencia del huésped, Yuen y Forbes (2009) modificaron la escala de resistencia existente (Hansen et al., 2005) para hacer una escala simple, una escala de intervalos de fácil aplicación. En la nueva escala, los valores aumentan con el aumento de los niveles de la enfermedad, y por lo tanto, la referencia es a la susceptibilidad en lugar de a la resistencia (es decir, los números más altos indican una mayor susceptibilidad). En contraste, muchas escalas de resistencia convencionales cuantifican el aumento de los niveles de resistencia como una función de la disminución de niveles de gravedad de la enfermedad. La dificultad con este enfoque es que la resistencia con valor cero, no es fácil de medir, ya que generalmente no se conoce el mayor nivel posible de susceptibilidad. Disponiendo un número bajo para la resistencia se resuelve este problema debido a que el nivel absoluto de la resistencia es simplemente la ausencia de enfermedad. 
Los avances en las TI (Tecnologías de la información) y en particular un mayor acceso a Internet ofrecen un nuevo potencial para la colaboración en los países en desarrollo. En el ámbito de la normalización y el manejo de datos, el CIP junto con algunos socios promueve el uso de las herramientas de introducción de datos que ayudarán a estandarizarlos y a proporcionar una mejor calidad de los mismos. Una de estas herramientas es DataCollector, una plantilla de Excel que ayuda a estandarizar y mantener la calidad de los datos ingresados, pero también tiene otras características tales como el desarrollo de los libros de campo y el análisis de los datos. DataCollector realiza el análisis de la estabilidad multilocacional y calcula la escala numérica de susceptibilidad al TTP descrito anteriormente(Simon et al., 2012). La determinación de la estabilidad de la resistencia a través de localidades sólo se puede mejorar con una mejor colaboración entre los investigadores de la papa. Con este fin, se han promovido redes de investigación durante muchos años. En el momento de escribir este artículo, existen redes activas de papa en Latinoamérica y en el Sudeste de Asia, y también existían en años anteriores en el África Subsahariana. Las redes son especialmente buenas en la promoción de la transferencia horizontal de conocimientos. Por ejemplo, los datos de los ensayos de cultivares en un país pueden guiar la selección de cultivares candidatos para otros países. El papel del monitoreo de patógenos en la búsqueda de resistencia duradera también requiere de una planificación y coordinación del muestreo y la evaluación de cepas; de lo contrario, los datos no se pueden comparar con facilidad a través de las localidades. En Europa se formaron redes de investigadores: EU.NET.ICP se estableció en 1994, seguido por Eucablight en 2003; las dos redes se fusionaron en 2006 formando Euroblight (www.euroblight.org). Se realizó un estudio detallado para revisar la repetibilidad del fenotipado para la virulencia del patógeno a través de un número de laboratorios dentro Euroblight (Andrivon et al., 2011). Más recientemente, se formó una red de investigadores del TTP similar en América del Norte (http://usablight.org/). CIP y una serie de organizaciones 
asociadas formaron una red mundial llamada Iniciativa Global para el Tizón Tardío (GILB) en 1996 (Huarte et al., 2002; Forbes, 2009). GILB ha participado activamente en el intercambio de conocimientos y organizado tres conferencias mundiales sobre el TTP. Recientemente, GILB está inactiva debido a la falta de recursos financieros. Durante el XXVI Congreso de la Asociación Latinoamericana de la Papa (ALAP) en 2014 se conformará la Red Latina del Tizón Tardío. Con el financiamiento, se podría rápidamente construir un nuevo monitoreo mundial del sistema patógeno TTP utilizando la tecnología desarrollada para Rust Mapper en cereales (http://apps.cimmyt.org/gis/rustmapper/index.htm)..

\section{Contribución de los interesados en la selección y el desarrollo de cultivares}

El sector de la I + D de los países en desarrollo necesitará innovar institucionalmente. Por ejemplo, la relación de trabajo tradicional bilateral entre un centro internacional como el CIP y el programa nacional de papa de cada país está dando paso a un enfoque de cadena mucho más amplio involucrando al sector privado (productores de semillas, compradores y distribuidores, minoristas, etc), a las universidades, a grupos de agricultores, a grupos comunitarios y a organizaciones no gubernamentales (Thiele et al., 2011). La participación de un gran número de actores de la cadena de producción-comercialización aumentaría la probabilidad de seleccionar cultivares que cumplan las necesidades de todos. Algunas de las relaciones innovadoras necesarias no son simples; por ejemplo, encontrar un terreno común con la industria privada en el desarrollo de cultivares necesita de acuerdos adecuados sobre los derechos de propiedad. Un objetivo importante de cualquier sistema de producción y distribución de semillas es acortar el tiempo requerido para hacer semilla de nuevos cultivares disponibles para los agricultores. La papa tiene una tasa de multiplicación muy baja (alrededor de $10 \times$ por temporada) y la producción de semilla de un nuevo cultivar, por tanto, requiere de varias temporadas. Cuando se han utilizado sistemas de semillas formales, la 
introducción de nuevos cultivares puede ocurrir más rápidamente. Antes de 1980, sólo los cultivares europeos muy antiguos eran cultivados en el África Subsahariana. En los inicios de la década de 1980, se introdujeron sistemas de semillas simples en Ruanda y Burundi lo que ayudó a multiplicar los cultivares recién seleccionados. Esto condujo a una casi total renovación de los cultivares en Burundi, Ruanda y la República Democrática Oriental del Congo (Potts et al., 1985; Vander Zaag, 1982). Uno de los cultivares introducidos en ese momento, Cruza 148, todavía se cultiva ampliamente en estos países y mantiene un alto nivel de resistencia al TTP (Tabla 1). En la mayoría de los casos, los sistemas de semillas formales en los países en desarrollo son pequeños y producen un porcentaje mínimo de la semilla utilizada cada año. En la medida en que sean operativos, a menudo multiplican sólo los cultivares más cultivados y son poco eficaces en la distribución de cultivares recién desarrollados. Por esta razón, se necesitan formas creativas de distribución de nuevos cultivares. Un ejemplo es la distribución en red desarrollada por la ONG FIPS África (http://fipsafrica.org/) que entrega pequeñas cantidades de semilla limpia de nuevos cultivares (de muchos cultivos, incluida la papa) e información sobre el manejo del cultivo.

\section{Consideraciones finales}

La construcción de un futuro en el que los agricultores tengan acceso a cultivares de papa con altos niveles de resistencia duradera al TTP requerirá mejoras en los enfoques mencionados. Se espera una mayor comprensión de la genética de la resistencia y de la patogenicidad lo que puede llevar a la identificación de fuentes de resistencia duradera que se puedan combinar con los caracteres requeridos por la cadena de valor de la papa. Esta tarea se vería facilitada por un mayor conocimiento del patógeno, la evolución y seguimiento del cambio del patógeno, el uso común de procedimientos estandarizados, y la medición exacta de la resistencia a través de medidas y procedimientos estadísticos apropiados. La colaboración entre los distintos 
países o regiones a lo largo del cadena de valor de la papa permitiría una identificación más rápida de cultivares con amplia aceptabilidad. La difusión de nuevos cultivares debe ocurrir más rápido con sistemas de semillas mejorados que incorporan nuevos cultivares u otros enfoques innovadores en la distribución de semillas.

\section{Conflictos de interés}

Los autores declaran que no existen conflictos de interés.

\section{Literatura citada}

Andrade-Piedra, JL; P.A. Cáceres; M. Pumisacho; G. A. Forbes. 2009. Humans: the neglected corner of the disease tetrahedron-developing a training guide for resource-poor farmers to control potato late blight. Acta Hort 834:111-122

Andrivon, D.; J. Avendaño-Córcoles; A. M. Cameron. 2011 Stability and variability of virulence of Phytophthora infestans assessed in a ring test across European laboratories. Plant Path 60:556-565

Bofu, S.; T. Weiming; W. Jimin; W. Chunlin; Y. Zhengui; W. Shengwu; M. Huarte. 1996. Economic Impact of CIP-24 in China. In: Case studies of the Economic Impact of CIPRelated Technologies. T.S. Walker and C.C. Crissman (eds.). 158 pp. Lima, Perú: CIP: p 3150. ISBN 9290601817

Bourke, A. 1993. The visitation of God? The potato and the great Irish famine. Lilliput Press, Ltd., Dublin.

Bradshaw, J.E.; R.L. Wastie; H.E. Stewart; G.R. Mackay. 1995. Breeding for resistance to late blight in Scotland. In: Dowley LJ, Bannon E, Cooke LR et al (eds) Phytophthora infestans 150. Boole, Dublin, pp 246-253

Carrasco, E.; N. Estrada; J. Gabriel; G. Alfaro; Y. Larondelle; W. García; O. Quiroga. 1997. Seis Cultivares Potenciales de Papa con Resistencia al Tizón Tardío (Phytophthora infestans) en Bolivia. Revista Latinoamericana de la Papa. (1997). 9/10:106-122 
Cooke, L.; A.K. Lees. 2004. Markers, old and new, for examining Phytophthora infestans diversity. Pl Path 53:692-704

Cooke, L.; H. Schepers; A. Hermansen; R.A. Bain; N.J. Bradshaw; F. Ritchie; D.S. Shaw; A. Evenhuis; G.J.T. Kessel, J.G.N. Wander. 2011. Epidemiology and integrated control of potato late blight in Europe. Potato Res 54:183-222. doi:10.1007/s11540-011-9187-0

Estrada, N.; E. Fernández-Northcote; E. Carrasco; O. Navia. 1994. Mejoramiento genético para resistencia a enfermedades y plagas de la papa en Bolivia. In: Memorias del 1er Taller sobre Resistencia Duradera en Cultivos Alto Andinos de Bolivia, Colombia, Ecuador y Perú. Quito, Ecuador. p.111

Forbes, G.A.; M. G. Chacón; H.G. Kirk; M. A. Huarte; M. Van Damme; S. Distel; G. R. Mackay; H. E. Stewart; R. Lowe; J. M. Duncan; H. S. Mayton; W. E. Fry; D. Andrivon; D. Ellissèche; R. Pellé; H. W. Platt; G. MacKenzie; T. R. Tarn; L. T. Colon; D. J. Budding; H. Lozoya-Saldaña; A. Hernandez-Vilchis; S. Capezio. 2005. Stability of resistance to Phytophthora infestans in potato: an international evaluation. Plant Path 54:364-372

Forbes, G.A.; J.A. Landeo.2006. Late blight. In: Gopal J, P KSM (eds) Handbook of potato production, improvement, and postharvest management. Haworth, Binghamton, pp 279-314

Forbes, G. A. 2009. Late blight in developing countries and the role of the Global Initiative on Late Blight GILB. Proc Eleventh Euroblight Workshop. Applied Plant Research, Wageningen, pp 37-43

Fry, W. 2008. Phytophthora infestans: the plant (and R gene) destroyer. Molecular Plant Pathology 9:385-402

Forbes, G. A., 2012. Using host resistance to manage potato late blight with particular reference to developing countries. Potato Research 55:205-216

Gabriel, J.; G. Plata; X. Cadima; J. Franco. 2013. Solanum phureja Juz et Buk.: Valuable Source of Genetic Resistance to Potato Late Blight [Phytophthora infestans (Mont.) de Bary]. Revista Latinoamericana de la Papa. (17) 2:131-142 
Garrett, KA; R.J. Nelson; C. C. Mundt. 2001. The effects of host diversity and other management components on epidemics of potato late blight in the humid highland tropics. Phytopath 91:993-1000

Gómez-Alpizar, L.; I. Carbone; J.B. Ristaino. 2007. An Andean origin of Phytophthora infestans inferred from mitochondrial and nuclear gene genealogies. PANS 104:3306

Grünwald, N.J.; M.A. Cadena Hinojosa; O.R. Covarrubias. 2002. Potato cultivars from the Mexican national program: sources and durability of resistance against late blight. Phytopathology 92:688-693

Haas, B.J.; S. Kamoun; M.C. Zody; R.H.Y. Jiang; R.E. Handsaker; L.M. Cano; M. Grabherr. 2009. Genome sequence and analysis of the Irish potato famine pathogen Phytophthora infestans. Nature 461(7262): 393-398

Hansen, J.G.; M. Koppel; A. Valskyte. 2005. Evaluation of foliar resistance in potato to Phytophthora infestans based on an international field trial network. Pl Path 54:169-179

Haverkort, A.J.; P.C. Struik; R.G.F. Visser; E. Jacobsen. 2009. Applied biotechnology to combat late blight in potato caused by Phytophthora infestans. Potato Res 52:249-264. Hijmans, R.J.; G.A. Forbes; T.S. Walker. 2000. Estimating the global severity of potato late blight with GISlinked disease forecast models. Plant Path 49:697-705

Huarte, M.; W. Collins; C. Lizarraga, 2002. GILB, Iniciativa Global para el Tizón Tardío. In: Centro Internacional de la Papa, 2002. In: E.N. Fernandez-Northcote (ed). Memorias del Taller Internacional Complementando la Resistencia al Tizón (Phytophthora infestans) en los Andes, 2002, febrero13-16, 2001, Cochabamba, Bolivia, GILB, Taller Latinoamérica 1, Lima, Perú. ii-iv. ISBN 9290602139

Kromann, P.; W. Pradel; D. Cole; A. Taipe; G.A. Forbes. 2011. Use of the environmental impact quotient to estimate health and environmental impacts of pesticide usage in Peruvian and Ecuadorian potato producción.J Env Prot 02:581-591.doi:10.4236/jep.2011.25067

Lammerts van Bueren, E.T.; M. Tiemens-Hulscher; P.C .Struik. 2008. Cisgenesis does not solve the late blight problem of organic potato production: alternative breeding strategies. Potato Res 51:89-99 
Li, C.; J. Wang; D.H. Chien; E. Chujoy; B. Song; P. VanderZaag. 2010. Cooperation-88: a high yielding, multipurpose, late blight resistant cultivar growing in southwest China. Am J Potato Res.

Micheletto, S.; R. Boland; M. Huarte. 2000. Argentine wild diploid Solanum species as sources of quantitative late blight resistance. Theoretical and Applied Genetics 101 (5): 902906

Morales, R. 2001. Frecuencia de Aplicaciones del Fungicida CloratoloniI 82.5 para el Manejo de Phytophthora infestans en Tres Variedades de Papa. Revista Latinoamericana de la Papa. 12:49-56 49

Navia, O.; A. Gandarillas; E. N. Fernández-Northcote. (2001) Estrategias de Integración de Resistencia y Control Químico del Tizón de la Papa (Phytophthora infestans): Validación con Instituciones. Revista Latinoamericana de la Papa. 12:152-168

Nowicki, M.; M.R. Foolad; M. Nowakowska; E.U. Kozik. 2012. Potato and tomato late blight caused by Phytophthora infestans: an overview of pathology and resistance breeding. Plant Dis 96:4-17

Nyankanga, R.O.; H.C Wien; O.M. Olanya; P.S. Ojiambo. 2004. Farmers' cultural practices and management of potato late blight in Kenya highlands: implications for development of integrated disease management. Int J Pest Mang 50:135-144

Orozco, F.A.; D.C. Cole; G.A. Forbes; J. Kroschel; S. Wanigaratne; D. Arica. 2009. Monitoring adherence to the International FAO Code of Conduct on the Distribution and Use of Pesticides-highly hazardous pesticides in central Andean agriculture and farmers' rights to health. Int J Occ Env Health 15:255-268

Ortiz, O.; P. Winters; H. Fano. 1999. La Percepción de los Agricultores sobre el Problema del Tizón Tardío o Rancha (Phytophthora infestans) su Manejo: Estudio de Casos en Cajamarca, Perú. Revista Latinoamericana de la Papa. 11:97-120 97

Potts, A.L.; L. Kayitare; M.J. Potts. 1985. Atlas des variétés de pomme de terre diffusées au Burundi. ISABU, Dept. des Productions Vegetales, Bujumbura 
Robinson, R.A.1996. Return to resistance: breeding crops to reduce pesticide dependence. agAccess, Davis, CA, p 480

Salaman. R. 1989. The history and social influence of the potato. Cambridge University Press, Cambridge

Simon, R.; E. Salas; R. Eyzaguirre; H. Vilma; S. De Haan; M. Bonierbale. 2012. Desarrollo de un "software estadistico" para estandarización y chequeo de calidad de datos del campo en raíces y tubérculos (GDET4RT) para el mejoramiento de cultivos. 12th Int.l Conf., ALAP, Uberlandia Brazil

Stewart, H.E.; J.E. Bradshaw; B. Pande. 2003. The effect of the presence of R-genes for resistance to late blight (Phytophthora infestans) of potato (Solanum tuberosum) on the underlying level of field resistance. Plant Path 52:193-198

Tan, M.Y.A.; R.C.B. Hutten; R.G.F. Visser; H.J. van Eck. 2010. The effect of pyramiding Phytophthora infestans resistance genes R Pi-mcd1 and R Pi-ber in potato. T.A.Gen 121:117125.

Thiele, G.; A. Devaux; I. Reinoso; H. Pico; F. Montesdeoca; M. Pumisacho; J. AndradePiedra; C. Velasco; P. Flores; R. Esprella; A. Thomann; K. Manrique; D. Horton. 2011. Multi-stakeholder platforms for linking small farmers to value chains: evidence from the Andes. Int J Agr S. 9:423 433.

Trognitz, B. R.; M. Bonierbale; J.A. Landeo; G. Forbes; J.E. Bradshaw; G.R. Mackay; R. Waugh; M.A. Huarte; L. Colon. 2001. Improving Potato resistance to Disease under the Global Initiative on Late Blight In: Ed. D. Cooper, T. Hodkin and C. Spillane, Broadening the Genetic Base of Crop Production, FAO (Roma), p 385-398. ISBN 0-85199-411-3

Umaerus, V.; M. Umaerus; L. Erjefält; B.A. Nilsson. 1983. Control of Phytophthora by host resistance: problems and progress. In: Erwin DC, Bartinicki-Garcia S, Tsao PH (eds) Phytophthora its biology, taxonomy, ecology and pathology. APS Press, St. Paul, pp 315-327

Van der Zaag, P. 1982. Strategy for developing a national potato program for Rwanda. Root crops E Afr. IDRC, Ottawa, pp 39-44 
Vanderplank, J.E. 1956. Horizontal (polygenic) and vertical (oligogenic) resistance against blight. Am Potato J 42:306

Villamon, F.G.; D.M. Spooner; M. Orrillo. 2005. Late blight resistance linkages in a novel cross of the wild potato species Solanum paucissectum (series Piurana). Theor Appl Gen 111:1201-1214

Visker, M. 2005. Association between late blight resistance and foliage maturity type in potato: physiological and genetic studies. Dissertation, Wageningen Agricultural University

Vleeshouwers. V.G.A.A.; R. Finkers; D. Budding, M. Visser; M.M.J. Jacobs; R. van Berloo; M. Pel; N. Champouret; E. Bakker; P. Krenek; H. Rietman; D. Huigen; R. Hoekstra; A. Goverse; B. Vosman; E. Jacobsen; R.G.F. Visser. 2011. SolRgene: an online database to explore disease resistance genes in tuber-bearing Solanum species. BMC P1 Bio 11:116

Walker, T.S.; P.E. Schmiediche; R.J. Hijmans. 1999. World trends and patterns in the potato crop: an economic and geographic survey. Potato Res 42:241-364

Wastie, R.L. 1991. Breeding for resistance. Phytophthora infestans: the cause of late blight of potato. Academic, San Diego, pp 193-224

Yuen, J.E.; G.A. Forbes. 2009. Estimating the level of susceptibility to Phytophthora infestans in potato genotipos.Phytopathology 99:783-786

Yuen, J. 2012. Modelling pathogen competition and displacement-Phytophthora infestans in Scandinavia. Eur J Plant Pathol 133:25-32. doi:10.1007/s10658-011-9933-9 